\title{
Nakagami High-Order Statistics from a Different Perspective
}

\author{
José Cândido Silveira Santos Filho and Michel Daoud Yacoub
}

\begin{abstract}
The Nakagami fading signal has been traditionally modeled as a sum of multipath Rayleigh components. Recently, an alternative Nakagami model, particularly attractive for simulation, has been proposed in the literature: a single Rayleigh signal propagating over a nonlinear path. Here, we derive many important high-order statistics associated to this model (second-order distribution, autocorrelation, joint distribution of the envelope and its time derivative, average outage rate, and average outage duration) and compare these statistics to those of the traditional model, widely assigned to Nakagami fading. We show that the two models lead to very different highorder statistics, except for the autocorrelations, which are indeed similar.
\end{abstract}

Keywords - High-order statistics, Nakagami fading, nonlinear channels, simulation.

\section{INTRODUCTION}

In [1, Eq. (3)], Nakagami reported that the radiowave amplitude variations due to multipath fading can be well described by the probability density function (PDF)

$$
f_{N}(n)=\frac{2 m^{m} n^{2 m-1} \exp \left(-\frac{m n^{2}}{\Omega}\right)}{\Omega^{m} \Gamma(m)}, m \geq \frac{1}{2},
$$

where $N$ denotes the Nakagami fading envelope, $\Omega=E\left[N^{2}\right]$ is the mean power, $m=E\left[N^{2}\right] / V\left[N^{2}\right]$ is the fading parameter, and $\Gamma(\cdot)$ is the gamma function. $(E[\cdot]$ denotes expectation, $V[\cdot]$ variance.) The corresponding cumulative distribution function $(\mathrm{CDF})$ is

$$
F_{N}(n)=1-\frac{\Gamma\left(m, \frac{m n^{2}}{\Omega}\right)}{\Gamma(m)}, m \geq \frac{1}{2},
$$

where $\Gamma(\cdot, \cdot)$ is the incomplete gamma function.

Although Nakagami inferred (1) by inspecting measured fading data, he demonstrated in [1] that his distribution is indeed a general approximate solution to the so-called problem of random interference - the main cause of multipath fading. In such an interference scenario, it is reasonable to expect that the signal at the receiver arises a sum of multipath wave components that traveled on different paths from the transmitter. The resulting fading envelope can be then written as [1, Eq. (41)]

$$
\left|\sum_{i} A_{i} \exp \left(j \Theta_{i}\right)\right|
$$

where $A_{i}$ and $\Theta_{i}$ denote the envelope and the phase of the $i$ th component, respectively. Of course, the exact PDF

The authors are with the Department of Communications, School of Electrical and Computer Engineering, University of Campinas, PO Box 6101, 13083-852 Campinas, SP, Brazil, Tel: +55 (19) 3788 5106, Fax: +55 (19) 3289 1395, E-mail: $\{$ candido,michel $\} @$ wisstek.org. of (3) depends on the envelope and phase PDFs of each component. However, Nakagami showed that this PDF may be in general well approximated by (1) [1, Eqs. (42)-(46)]. His approximation has gained widespread use because of its mathematical ease, great flexibility, and, more important, good fit to empirical fading data [2], [3].

On the other hand, the simulation of the Nakagami fading channel is rather controversial. For other fading conditions such as Rayleigh, Rice, and Hoyt, the channel model itself can be directly used as a simulation scheme. For Nakagami fading, however, this approach no longer works. Since the Nakagami PDF and its underlying channel model only approximate each other, using (3) as a simulation scheme would not produce samples that follow (1) in a exact manner.

Many artifices have been proposed in the literature to produce Nakagami envelope samples. A classical method, suggested by Nakagami himself [1, Eqs. (63)-(64)], is to write the Nakagami process $N_{c}(t)$ ( $c$ stands for classical method) as the square root of the sum of $m$ i.i.d. squared Rayleigh processes $R_{i}(t)$, that is

$$
N_{c}(t)=\sqrt{\sum_{i=1}^{m} R_{i}^{2}(t)}
$$

where $t$ is a time index. The mean power of each Rayleigh process is $\Omega / m$. It is easy to show that (4) perfectly matches the Nakagami PDF. Indeed, many high-order statistics widely associated to the Nakagami envelope have been derived under the assumption of (4), including second-order PDF, autocorrelation, joint PDF of the channel envelope and its time derivative, average outage rate, and average outage duration. These statistics shall be revisited in the next section. The main limitation of the classical method is that it applies to $m$ integer only ${ }^{1}$.

Another attractive method was recently proposed that allows for $m$ real [3]. In this method, the Nakagami signal is thought of as a single Rayleigh signal that propagates over a memoryless nonlinear path. In other words, the Nakagami samples are generated from Rayleigh samples by using the standard percentile transformation method, also called inversion method [4, Eq. (7-157)]. This approach connects the Nakagami process $N_{i}(t)$ ( $i$ stands for inversion method) to the Rayleigh one $R(t)$ through the CDFs of each, as follows [3, Fig. 3]

$$
N_{i}(t)=F_{N}^{-1}\left(F_{R}(R(t))\right) \triangleq g(R(t)),
$$

where $F_{N}^{-1}(\cdot)$ is the inverse Nakagami CDF and $F_{R}(\cdot)$ is the

\footnotetext{
${ }^{1}$ In fact, (4) can be relaxed to allow for $m$ half-integer [2].
} 
unit-power Rayleigh $\mathrm{CDF}^{2}$

$$
F_{R}(r)=1-\exp \left(-r^{2}\right) .
$$

The inverse Nakagami CDF required in (5) is unknown in closed form and must be numerically approximated. An accurate approximation is presented in [3]. In the remaining of the text, we call (5) the inversion method, as opposed to the classical method in (4).

Other methods exist for simulating Nakagami fading [5][8], but we shall not address them here. Our aim is to derive important high-order statistics associated to the Nakagami fading channel as implemented by the inversion methodsecond-order PDF, autocorrelation, joint PDF of the envelope and its time derivative, average outage rate (AOR), and average outage duration (AOD) - and to compare these statistics to those of the classical method, extensively assigned to $\mathrm{Na}$ kagami fading. We show that the two methods lead to very different high-order statistics, except for the autocorrelation, which are indeed similar.

In the following, the time index $t$ is sometimes omitted to alleviate the notation.

\section{The Classical Method Revisited}

As mentioned before, it is easy to show that the PDF $f_{N_{c}}(\cdot)$ and $\mathrm{CDF} F_{N_{c}}(\cdot)$ of $N_{c}$ are given by

$$
f_{N_{c}}(n)=f_{N}(n) \text { and } F_{N_{c}}(n)=F_{N}(n),
$$

where $f_{N}(\cdot)$ is the Nakagami PDF and $F_{N}(\cdot)$ is the Nakagami $\mathrm{CDF}$, presented in (1) and (2). Important high-order statistics of the process $N_{c}(t)$ have been investigated in the literature. Next, we reproduce some of them.

It has been shown in [2] that for isotropic scattering the time derivative $\dot{N}_{c}(t)$ of $N_{c}(t)$ is Gaussian distributed with zero mean and variance

$$
\dot{\sigma}_{c}^{2}=\pi^{2} f_{D}^{2} \Omega / m,
$$

where $f_{D}$ is the maximum Doppler shift in Hz. Accordingly,

$$
f_{\dot{N}_{c}}(\dot{n})=\frac{\exp \left(-\frac{\dot{n}^{2}}{2 \dot{\sigma}_{c}^{2}}\right)}{\sqrt{2 \pi} \dot{\sigma}_{c}} .
$$

It has also been shown in [2] that $N_{c}(t)$ and $\dot{N}_{c}(t)$ are independent variates ${ }^{3}$, so that

$$
f_{\dot{N}_{c} \mid N_{c}}(\dot{n} \mid n)=f_{\dot{N}_{c}}(\dot{n})
$$

and

$$
\begin{aligned}
f_{\dot{N}_{c}, N_{c}}(\dot{n}, n) & =f_{\dot{N}_{c}}(\dot{n}) f_{N_{c}}(n) \\
& =\frac{\sqrt{2} m^{m} n^{2 m-1} \exp \left(-\frac{m n^{2}}{\Omega}-\frac{\dot{n}^{2}}{2 \dot{\sigma}_{c}^{2}}\right)}{\sqrt{\pi} \dot{\sigma}_{c} \Omega^{m} \Gamma(m)}(11)
\end{aligned}
$$

The second-order PDF of $N_{c}(t)$ is also of interest. From (4),

\footnotetext{
${ }^{2}$ For convenience, we have normalized the Rayleigh process to have unit mean power.

${ }^{3}$ It does not imply that $N_{c}\left(t_{1}\right)$ and $\dot{N}_{c}\left(t_{2}\right)$ are still independent for $t_{1} \neq t_{2}$ or, equivalently, that $N_{c}(t)$ and $\dot{N}_{c}(t)$ are independent random processes.
}

this PDF is obtained as [1, Eq. 126]

$$
\begin{gathered}
f_{N_{c}(t), N_{c}(t+\tau)}\left(n_{1}, n_{2}\right)=\frac{4 m^{m+1}\left(n_{1} n_{2}\right)^{m}}{\Omega^{m+1} \rho_{2}(\tau)^{\frac{m-1}{2}}\left(1-\rho_{2}(\tau)\right) \Gamma(m)} \\
\times \exp \left(-\frac{m\left(n_{1}^{2}+n_{2}^{2}\right)}{\Omega\left(1-\rho_{2}(\tau)\right)}\right) I_{m-1}\left(m-1, \frac{2 \sqrt{\rho_{2}(\tau)} m n_{1} n_{2}}{\Omega\left(1-\rho_{2}(\tau)\right)}\right),
\end{gathered}
$$

where $I_{\nu}(\cdot)$ is the modified Bessel function of first kind and $\nu$ th order, and $\rho_{2}(\tau)$ is the power correlation coefficient of each underlying Rayleigh process $R_{i}(t)$. Expressions for $\rho_{2}(\tau)$ have been extensively reported in the literature for isotropic as well as anisotropic fading scenarios [9], [10]. It can be shown that $\rho_{2}(\tau)$ is also the power correlation coefficient of $N_{c}(t)[1$, Eq. 127].

From (12), the autocorrelation function (ACF) $A_{N_{c}}(\tau)$ and the autocorrelation coefficient (ACC) $\rho_{N_{c}}(\tau)$ of $N_{c}(t)$ can be derived as [1, Eqs. (137) and (139)]

$$
\begin{aligned}
& A_{N_{c}}(\tau)=\frac{\Omega \Gamma^{2}\left(m+\frac{1}{2}\right)}{m \Gamma^{2}(m)}{ }_{2} F_{1}\left(-\frac{1}{2},-\frac{1}{2} ; m ; \rho_{2}(\tau)\right) \\
& \rho_{N_{c}}(\tau)=\frac{\Gamma^{2}\left(m+\frac{1}{2}\right)\left({ }_{2} F_{1}\left(-\frac{1}{2},-\frac{1}{2} ; m ; \rho_{2}(\tau)\right)-1\right)}{\Gamma(m) \Gamma(m+1)-\Gamma^{2}\left(m+\frac{1}{2}\right)} .
\end{aligned}
$$

Other important dynamic metrics are the AOR $\mathcal{R}_{N_{c}}(\cdot)$ and AOD $\mathcal{D}_{N_{c}}(\cdot)$ of $N_{c}(t)$, which reflect the autocorrelation properties of the channel. They are given by [2, Eqs. (17) and (21)]

$$
\begin{aligned}
& \mathcal{R}_{N_{c}}(n)=\frac{\sqrt{2 \pi} f_{D} m^{m-\frac{1}{2}} n^{2 m-1} \exp \left(-\frac{m n^{2}}{\Omega}\right)}{\Gamma(m) \Omega^{m-\frac{1}{2}}} \\
& \mathcal{D}_{N_{c}}(n)=\frac{\Omega^{m-\frac{1}{2}}\left[\Gamma(m)-\Gamma\left(m, \frac{m n^{2}}{\Omega}\right)\right]}{\sqrt{2 \pi} f_{D} m^{m-\frac{1}{2}} n^{2 m-1} \exp \left(-\frac{m n^{2}}{\Omega}\right)} .
\end{aligned}
$$

\section{The InVERSion Method ANALYZED}

In this section, we derive the corresponding high-order statistics of the inversion method. To the best of our knowledge, all of the expressions derived here are new.

As desired, the PDF $f_{N_{i}}(\cdot)$ and $\operatorname{CDF} F_{N_{i}}(\cdot)$ of $N_{i}$ are those of a Nakagami variate, i.e.

$$
f_{N_{i}}(n)=f_{N}(n) \text { and } F_{N_{i}}(n)=F_{N}(n) .
$$

However, the high-order statistics of $N_{i}(t)$ differ from those of $N_{c}(t)$, as shown next.

For convenience, instead of (5), we shall use the inverse relation, given by

$$
R(t)=F_{R}^{-1}\left(F_{N}\left(N_{i}(t)\right)\right)=g^{-1}\left(N_{i}(t)\right) \triangleq h\left(N_{i}(t)\right),
$$

where $F_{R}^{-1}(\cdot)$ is the inverse unity-power Rayleigh CDF

$$
F_{R}^{-1}(x)=\sqrt{-\ln (1-x)} .
$$

The reason to use (18) instead of (5) is that $h(\cdot)$ can be obtained in closed form, whereas $g(\cdot)$ cannot. Indeed, using 
(2) and (19) into (18), $h(\cdot)$ is calculated as

$$
h(n)=\sqrt{-\ln \left(\frac{\Gamma\left(m, \frac{m n^{2}}{\Omega}\right)}{\Gamma(m)}\right)} .
$$

We begin by investigating the conditional PDF of the time derivative $\dot{N}_{i}(t)$ of $N_{i}(t)$, given $N_{i}(t)$. Differentiating (18) with respect to time, we obtain

$$
\dot{R}(t)=h^{\prime}\left(N_{i}(t)\right) \dot{N}_{i}(t),
$$

where $h^{\prime}(\cdot)$ is the first derivative of $h(\cdot)$, calculated as

$$
h^{\prime}(n)=\frac{m^{m} n^{2 m-1} \exp \left(-\frac{m n^{2}}{\Omega}\right)}{\Omega^{m} \Gamma\left(m, \frac{m n^{2}}{\Omega}\right) \sqrt{-\ln \left(\frac{\Gamma\left(m, \frac{m n^{2}}{\Omega}\right)}{\Gamma(m)}\right)}} .
$$

It is well known that for isotropic scattering $\dot{R}(t)$ and $R(t)$ are independent variates, and that $\dot{R}(t)$ is Gaussian distributed with zero mean and variance $\pi^{2} f_{D}^{2}$ [9], so that

$$
\begin{aligned}
f_{R}(r) & =2 r \exp \left(-r^{2}\right) \\
f_{\dot{R}}(\dot{r}) & =\frac{\exp \left(-\frac{\dot{r}^{2}}{2 \pi^{2} f_{D}^{2}}\right)}{\sqrt{2} \pi^{3 / 2} f_{D}} \\
f_{\dot{R}, R}(\dot{r}, r) & =f_{\dot{R}}(\dot{r}) f_{R}(r)=\frac{\sqrt{2} r \exp \left(-r^{2}-\frac{\dot{r}^{2}}{2 \pi^{2} f_{D}^{2}}\right)}{\pi^{3 / 2} f_{D}}
\end{aligned}
$$

Note in (5) that $N_{i}(t)$ is a memoryless function of $R(t)$ and thus also independent of $\dot{R}(t)$. Therefore, from (21), $\dot{N}_{i}(t)$ is Gaussian conditioned on $N_{i}(t)=n$, with zero mean and variance

$\dot{\sigma}_{i}^{2}(n)=\frac{\pi^{2} f_{D}^{2}}{h^{\prime 2}(n)}=-\frac{\pi^{2} f_{D}^{2} \Omega^{2 m} \Gamma^{2}\left(m, \frac{m n^{2}}{\Omega}\right) \ln \left(\frac{\Gamma\left(m, \frac{m n^{2}}{\Omega}\right)}{\Gamma(m)}\right)}{m^{2 m} n^{4 m-2} \exp \left(-\frac{2 m n^{2}}{\Omega}\right)}$

Accordingly,

$$
f_{\dot{N}_{i} \mid N_{i}}(\dot{n} \mid n)=\frac{\exp \left(-\frac{\dot{n}^{2}}{2 \dot{\sigma}_{i}^{2}(n)}\right)}{\sqrt{2 \pi} \dot{\sigma}_{i}(n)} .
$$

Note that $f_{\dot{N}_{i} \mid N_{i}}(\dot{n} \mid n)$ depends on $n$. That is, $N_{i}(t)$ and $\dot{N}_{i}(t)$ are dependent variates. The joint PDF of $N_{i}(t)$ and $\dot{N}_{i}(t)$ can be calculated from (25) by performing the transformation of variables defined in (18) and (21), which gives

$$
f_{\dot{N}_{i}, N_{i}}(\dot{n}, n)=h^{\prime 2}(n) f_{\dot{R}, R}\left(h^{\prime}(n) \dot{n}, h(n)\right),
$$

where $h^{\prime 2}(n)$ is the Jacobian of the transformation. Replacing (20), (22), and (25) into (28), and after some algebraic manipulations, we obtain

$$
\begin{aligned}
& f_{\dot{N}_{i}, N_{i}}(\dot{n}, n)=\frac{\sqrt{2} m^{2 m} n^{4 m-2}}{\pi^{3 / 2} f_{D} \Omega^{2 m} \Gamma(m) \Gamma\left(m, \frac{m n^{2}}{\Omega}\right)} \\
& \times \frac{\exp \left(-\frac{2 m n^{2}}{\Omega}+\frac{m^{2 m} n^{4 m-2} \exp \left(-\frac{2 m n^{2}}{\Omega}\right) \dot{n}^{2}}{2 \pi^{2} f_{D}^{2} \Omega^{2 m} \Gamma^{2}\left(m, \frac{m n^{2}}{\Omega}\right) \ln \left(\frac{\Gamma\left(m, \frac{m n^{2}}{\Omega}\right)}{\Gamma(m)}\right)}\right)}{\sqrt{-\ln \left(\frac{\Gamma\left(m, \frac{m n^{2}}{\Omega}\right)}{\Gamma(m)}\right)}} .
\end{aligned}
$$

The second-order PDF of the unit-power Rayleigh process $R(t)$ is given by (12) with $m=\Omega=1$, which reduces to

$f_{R(t), R(t+\tau)}\left(r_{1}, r_{2}\right)=\frac{4 r_{1} r_{2} \exp \left(-\frac{r_{1}^{2}+r_{2}^{2}}{1-\rho_{2}(\tau)}\right) I_{0}\left(\frac{2 \sqrt{\rho_{2}(\tau)} r_{1} r_{2}}{1-\rho_{2}(\tau)}\right)}{1-\rho_{2}(\tau)}$,

where $\rho_{2}(\tau)$ is the power autocorrelation coefficient of $R(t)$. By performing the transformation of variables defined in (18), the second-order PDF of $N_{i}(t)$ is obtained from (30) as

$$
\begin{aligned}
& f_{N_{i}(t), N_{i}(t+\tau)}\left(n_{1}, n_{2}\right)=\frac{4 m^{2 m}\left(n_{1} n_{2}\right)^{2 m-1}}{\Omega^{2 m}\left(1-\rho_{2}(\tau)\right) \Gamma(m)^{\frac{2}{1-\rho_{2}(\tau)}}} \\
\times & {\left[\Gamma\left(m, \frac{m n_{1}^{2}}{\Omega}\right) \Gamma\left(m, \frac{m n_{2}^{2}}{\Omega}\right)\right]^{\frac{\rho_{2}(\tau)}{1-\rho_{2}(\tau)}} \exp \left(-\frac{m\left(n_{1}^{2}+n_{2}^{2}\right)}{\Omega}\right) } \\
\times & I_{0}\left(\frac{2 \sqrt{\rho_{2}(\tau)}}{1-\rho_{2}(\tau)} \sqrt{\left.\ln \left(\frac{\Gamma\left(m, \frac{m n_{1}^{2}}{\Omega}\right)}{\Gamma(m)}\right) \ln \left(\frac{\Gamma\left(m, \frac{m n_{2}^{2}}{\Omega}\right)}{\Gamma(m)}\right)\right) .} .\right.
\end{aligned}
$$

By definition, the ACF $A_{N_{i}}(\tau)$ of $N_{i}(t)$ can be written from (5) as

$$
A_{N_{i}}(\tau)=\int_{0}^{\infty} \int_{0}^{\infty} g\left(r_{1}\right) g\left(r_{2}\right) f_{R(t), R(t+\tau)}\left(r_{1}, r_{2}\right) d r_{1} d r_{2},
$$

where $f_{R(t), R(t+\tau)}(\cdot, \cdot)$ is given in (30). However, as mentioned before, $g(\cdot)$ is unknown in closed form. This problem can be circumvented by the change of variables $n_{1}=g\left(r_{1}\right)$ and $n_{2}=g\left(r_{2}\right)$, yielding

$$
\begin{array}{r}
A_{N_{i}}(\tau)=\int_{0}^{\infty} \int_{0}^{\infty} n_{1} n_{2} f_{R(t), R(t+\tau)}\left(h\left(n_{1}\right), h\left(n_{2}\right)\right) \\
\times h^{\prime}\left(n_{1}\right) h^{\prime}\left(n_{2}\right) d n_{1} d n_{2},
\end{array}
$$

where $h(\cdot)$ and $h^{\prime}(\cdot)$ are given in (20) and (22), respectively. It seems that (33) has no closed-form solution, requiring numerical evaluation.

By definition, the ACC $\rho_{N_{i}}(\tau)$ of $N_{i}(t)$ is obtained as

$$
\rho_{N_{i}}(\tau)=\frac{A_{N_{i}}(\tau)-E\left[N_{i}(t)\right]^{2}}{V\left[N_{i}(t)\right]} .
$$

For convenience, one may use $\Omega=1$ in evaluating (34), for $\Omega$ has no impact on the ACC. Replacing $E\left[N_{i}(t)\right]$ [1, Eq. (17)] 
and $V\left[N_{i}(t)\right]$ [1, Eq. (18)] with $\Omega=1$ into (34), we obtain

$$
\rho_{N_{i}}(\tau)=\frac{\left.A_{N_{i}}(\tau)\right|_{\Omega=1}-\frac{\Gamma\left(m+\frac{1}{2}\right)^{2}}{m \Gamma(m)^{2}}}{1-\frac{\Gamma\left(m+\frac{1}{2}\right)^{2}}{m \Gamma(m)^{2}}},
$$

where $\left.A_{N_{i}}(\tau)\right|_{\Omega=1}$ has to be solved numerically as in (33).

In (18), $N_{i}(t)$ is mapped into $R(t)$ through a memoryless transformation, namely $h(\cdot)$. Thus, the process $N_{i}(t)$ crosses a given level $n$ at the same rate at which the process $R(t)$ crosses the level $h(n)$. In other words, the AOR $\mathcal{R}_{N_{i}}(n)$ of $N_{i}(t)$ can be obtained in terms of the $\mathrm{AOR} \mathcal{R}_{R}(r)$ of $R(t)$ as

$$
\mathcal{R}_{N_{i}}(n)=\mathcal{R}_{R}(h(n)) \text {. }
$$

The AOR of the unit-power Rayleigh process is given by (15) with $m=\Omega=1$, which reduces to

$$
\mathcal{R}_{R}(r)=\sqrt{2 \pi} f_{D} r \exp \left(-r^{2}\right) .
$$

Replacing (37) into (36) with use of (20), we have

$$
\mathcal{R}_{N_{i}}(n)=\frac{\sqrt{2 \pi} f_{D} \Gamma\left(m, \frac{m n^{2}}{\Omega}\right) \sqrt{-\ln \left(\frac{\Gamma\left(m, \frac{m n}{\Omega}\right)}{\Gamma(m)}\right)}}{\Gamma(m)} .
$$

Finally, the AOD $\mathcal{D}_{N_{i}}(n)$ of $N_{i}(t)$ is obtained as $F_{N_{i}}(n) / \mathcal{R}_{N_{i}}(n)$, which gives

$$
\mathcal{D}_{N_{i}(t)}(n)=\frac{\Gamma(m)-\Gamma\left(m, \frac{m n^{2}}{\Omega}\right)}{\sqrt{2 \pi} f_{D} \Gamma\left(m, \frac{m n^{2}}{\Omega}\right) \sqrt{-\ln \left(\frac{\Gamma\left(m, \frac{m n^{2}}{\Omega}\right)}{\Gamma(m)}\right)}} .
$$

\section{COMPARISONS}

We now compare the Nakagami high-order statistics derived for the inversion method with those of the classical method, usually assigned to Nakagami fading.

In the classical method, as widely known, the envelope time derivative is Gaussian and independent of the envelope. In the inversion method, as we showed, the envelope time derivative is neither Gaussian nor independent of the envelope. Fig. 1 shows the variance of $\dot{N}$ conditioned on $N$. This is given by (8) for the classical method, and by (26) for the inversion method. In the former, the variance of $\dot{N}$ is clearly insensitive to $N$. In the latter, however, the variance may change considerably with $N$, mainly for low levels, as observed from the curves. Fig. 2 shows the PDF of $\dot{N}$, given by (9) for the classical method, and by the numerical integration of (29) over $n$ for the inversion method. Note how for $m>1$ the PDF tails are much more pronounced in the inversion method, departing considerably from the Gaussian PDFs of the classical method.

The second-order PDFs for the classical and inversion methods are given by (12) and (31), respectively. We have used the Kullback-Leibler distance [11] to assess the dissimilarity between these two PDFs. Fig. 3 shows the corresponding results. Note that the Kullback-Leibler distance vanishes as $\rho_{2}(\tau) \rightarrow 0$. This is expected, because in such a case the Nakagami variates become independent, and the second-order

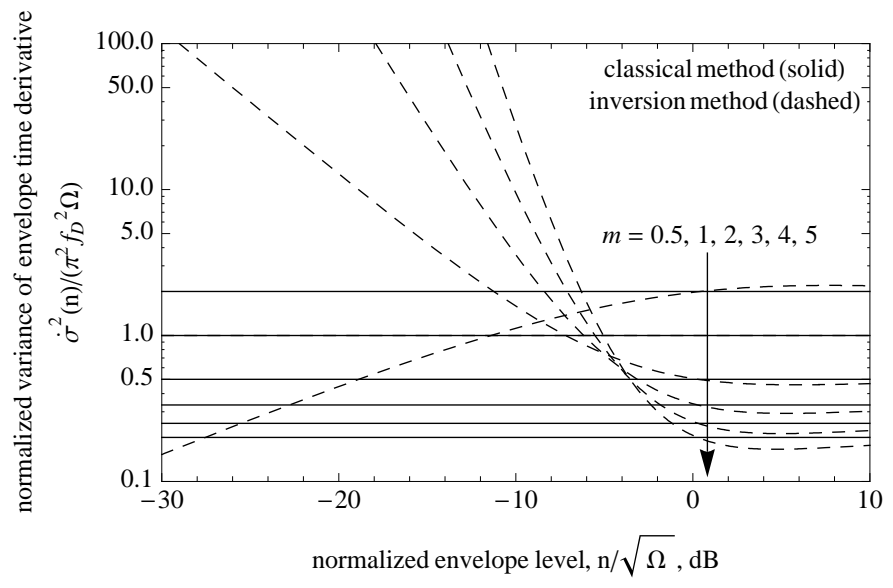

Fig. 1. Variance of the Nakagami envelope time derivative conditioned on the envelope level.

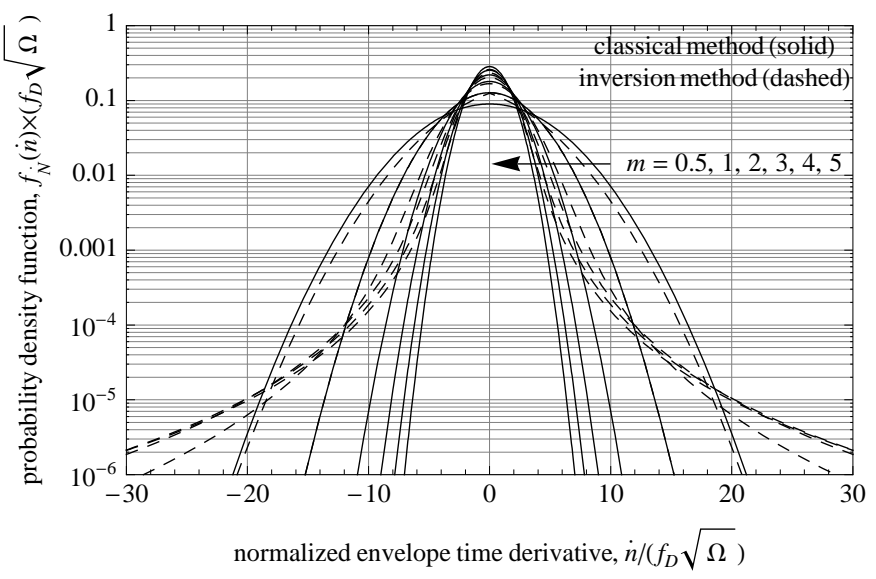

Fig. 2. PDF of the Nakagami envelope time derivative.

PDFs converge, in both methods, to the product of two Nakagami PDFs. On the other hand, the Kullback-Leibler distance increases steadily with $\rho_{2}(\tau)$, so that the two PDFs may differ considerably from each other for high correlation values. Take, for instance, $\rho_{2}(\tau)=0.9$. In this case, from the curves, the Kullback-Leibler distance is greater than 0.02 for any value of $m$. As a term of comparison, it can be shown that 0.02 is the same distance between a zero-mean, unit-variance Gaussian PDF and a zero-mean, 0.703-variance Gaussian PDF.

Although the second-order PDFs for the classical and inversion methods may differ considerably from each other, the corresponding ACFs (or ACCs) are quite similar, for any value of $m$. This is shown in Fig. 4, in which the Nakagami ACC is plotted against the underlying Rayleigh ACC. The Nakagami ACC is given by (14) for the classical method, and by (35) for the inversion method. The underlying Rayleigh ACC is given by (14) or (35) with $m=1$. Note that the difference between the ACCs of the two methods does not exceed $5 \times 10^{-2}$.

Figs. 5 and 6 show the impact of the inversion method on the AOR and AOD of the Nakagami envelope, respectively, as compared with those of the classical method. At low envelope levels, the inversion method reduces the AOR for $m<1$ and increases it for $m>1$. Correspondingly, the inversion method increases the AOD for $m<1$ and reduces it for 


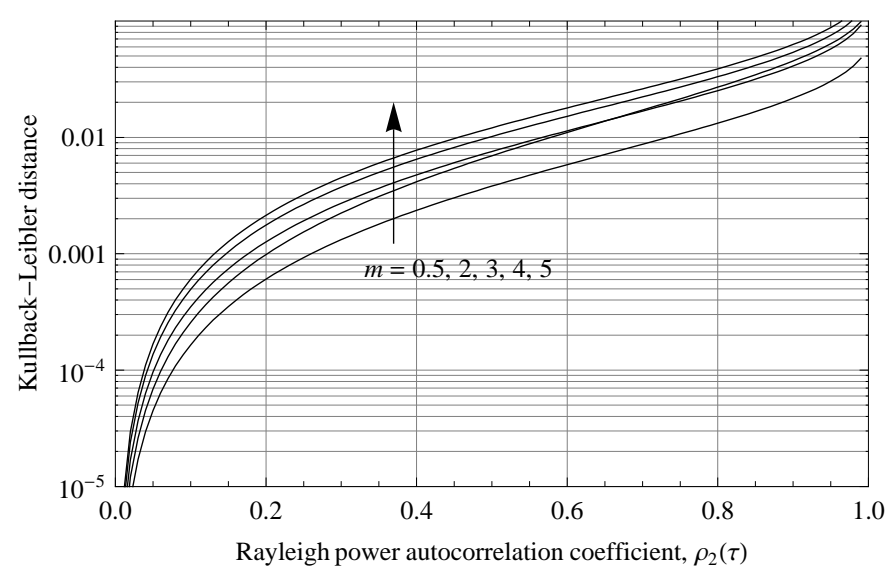

Fig. 3. Kullback-Leibler distance between the joint PDFs of the Nakagami envelope and its time derivative for the classical and inversion methods.

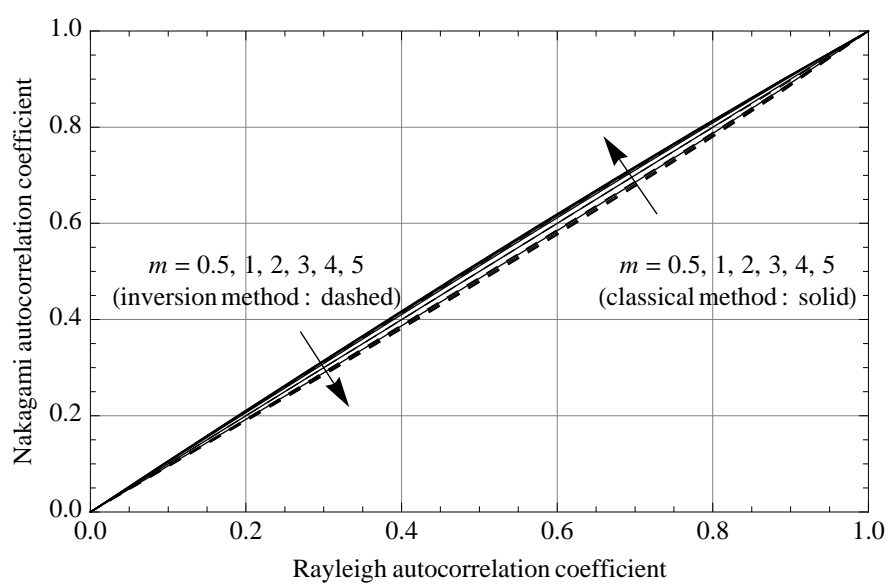

Fig. 4. Nakagami autocorrelation coefficient as a function of the underlying Rayleigh autocorrelation coefficient.

$m>1$. At high envelope levels, however, the AOR and AOD of the inversion method are observed to be practically indistinguishable from those of the classical method.

\section{CONCLUSIONS}

The modeling and simulation of Nakagami fading channels by the inversion method is certainly attractive, because it is simple and allows for $m$ real. However, as we showed here, the inversion method leads to high-order statistics that differ considerably from those of the classical method, commonly assigned to Nakagami fading.

\section{REFERENCES}

[1] M. Nakagami, "The $m$-distribution - a general formula of intensity distribution of rapid fading," in Statistical Methods in Radio Wave Propagation, W. C. Hoffman, Ed. Oxford, England: Pergamon, 1960.

[2] M. D. Yacoub, J. E. V. Bautista, and L. G. de Resende Guedes, "On higher order statistics of the Nakagami-m distribution," IEEE Trans. Veh. Technol., vol. 48, no. 3, pp. 790-794, May 1999.

[3] N. C. Beaulieu and C. Cheng, "Efficient Nakagami- $m$ fading channel simulation," IEEE Trans. Veh. Technol., vol. 54, no. 2, pp. 413-424, Mar. 2005.

[4] A. Papoulis and S. U. Pillai, Probability, Random Variables and Stochastic Processes, 4th ed. New York: McGraw-Hill, 2002.

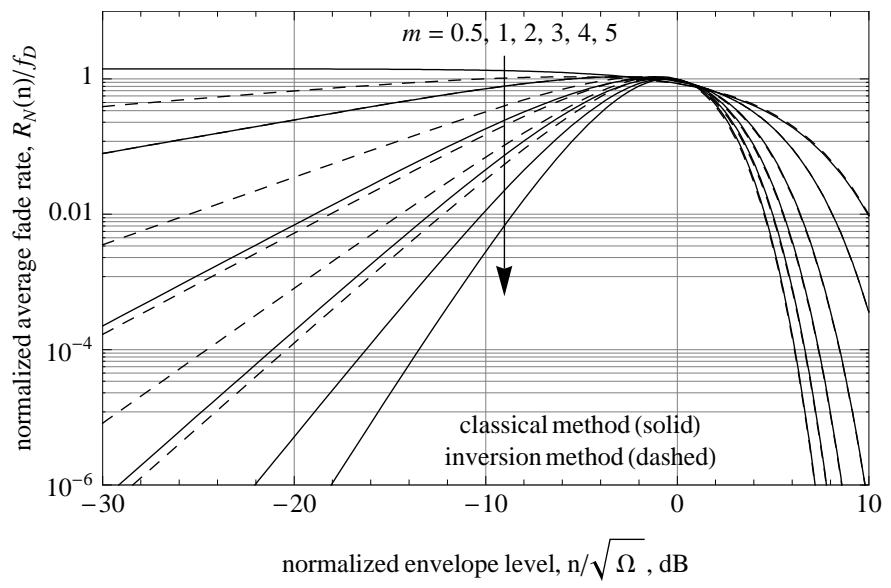

Fig. 5. Average outage rate of the Nakagami envelope.

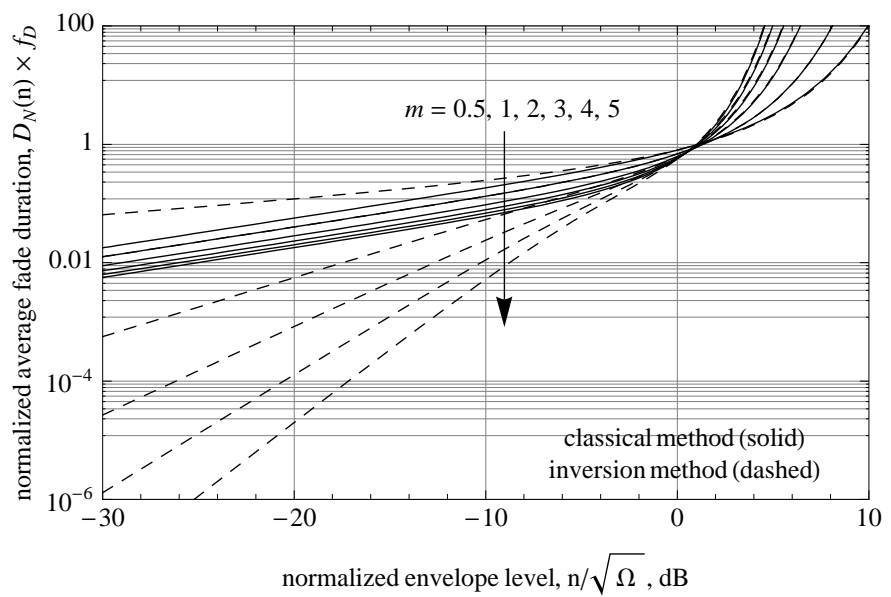

Fig. 6. Average outage duration of the Nakagami envelope.

[5] K. W. Yip and T. S. Ng, "A simulation model for Nakagami-m fading channels, $m<1$," IEEE Trans. Commun., vol. 48, no. 2, pp. 214-221, Feb. 2000.

[6] Q. T. Zhang, "A decomposition technique for efficient generation of correlated Nakagami fading channels," IEEE Journal Select. Areas Commun., vol. 18, no. 11, pp. 2385-2392, Nov. 2000.

[7] K. Zhang, Z. Song, and Y. L. Guan, "Simulation of Nakagami fading channels with arbitrary cross-correlation and fading parameters," IEEE Trans. Wireless Commun., vol. 3, no. 5, pp. 1463-1468, Sep. 2004.

[8] J. C. S. Santos Filho, M. D. Yacoub, and G. Fraidenraich, "A simple accurate method for generating autocorrelated Nakagami- $m$ envelope sequences," IEEE Commun. Lett., vol. 11, no. 3, pp. 231-233, Mar. 2007

[9] W. C. Jakes, Microwave Mobile Communications. New York: Wiley, 1974.

[10] A. Abdi, J. A. Barger, and M. Kaveh, "A parametric model for the distribution of the angle of arrival and the associated correlation function and power spectrum at the mobile station," IEEE Trans. Veh. Technol., vol. 51, no. 3, pp. 425-434, May 2002.

[11] T. M. Cover and J. A. Thomas, Elements of Information Theory. Wiley, 1991. 